Khattab et al., Afr J Tradit Complement Altern Med., (2017) 14 (4): 169-179

https://doi.org/10.21010/ajtcam.v14i4.20

\title{
EFFICIENCY OF BORAGE SEEDS OIL AGAINST GAMMA IRRADIATION-INDUCED HEPATOTOXICITY IN MALE RATS: POSSIBLE ANTIOXIDANT ACTIVITY
}

\author{
Hala A.H. Khattab ${ }^{1,2 *}$, Inas Z.A. Abdallah ${ }^{2}$, Fatimah M. Yousef ${ }^{1}$ and Etimad A. Huwait ${ }^{3}$ \\ ${ }^{1}$ Food and Nutrition Department, Faculty of Home Economics, King Abdulaziz University, Jeddah, Saudi \\ Arabia. ${ }^{2}$ Nutrition and Food Science Department, Faculty of Home Economics, Helwan University, Egypt. \\ ${ }^{3}$ Biochemistry Department, Faculty of Science, King Abdulaziz University, Jeddah, Saudi Arabia.
}

* Corresponding Author E-mail: hkhattab@kau.edu.sa, haya khattab@hotmail.com

\begin{abstract}
Background: Borage (Borago officinal L.) is an annual herbaceous plant of great interest because its oil contains a high percentage of $\gamma$-linolenic acid (GLA). The present work was carried out to detect fatty acids composition of the oil extracted from borage seeds (BO) and its potential effectiveness against $\gamma$-irradiation- induced hepatotoxicity in male rats.

Materials and Methods: GC-MS analysis of fatty acids methyl esters of BO was performed to identify fatty acids composition. Sixty rats were divided into five groups (12 rats each): Control, irradiated; rats were exposed to (6.5 Gy) of whole body $\gamma$-radiation, BO $(50 \mathrm{mg} / \mathrm{kg} \mathrm{b.wt})$, irradiated BO post-treated and irradiated BO prepost-treated. Six rats from each group were sacrificed at two time intervals 7 and 15 days post-irradiation. Serum aspartate aminotransferase (AST), alanine aminotransferase (ALT), gamma glutamyl transferase (GGT) levels, lipids profile, as well as serum and hepatic reduced glutathione (GSH) and lipid peroxide (malondialdehyde) (MDA) levels were assessed. Histopathological examination of liver sections were also carried out.

Results: The results showed that the high contents of BO extracted by cold pressing, were linoleic acid (34.23\%) and GLA $(24.79 \%)$. Also, oral administration of BO significantly improved serum levels of liver enzymes, lipids profile, as well as serum and hepatic GSH and MDA levels $(\mathrm{p}<0.001)$ as compared with irradiated rats after 15 days post irradiation. Moreover, it exerted marked amelioration against irradiation-induced histopathological changes in liver tissues. The improvement was more pronounced in irradiated BO prepost-treated group than irradiated BO post-treated. Conclusion: BO has a beneficial role in reducing hepatotoxicity and oxidative stress induced by radiation exposure. Therefore, BO may be used as a beneficial supplement for patients during radiotherapy treatment.
\end{abstract}

Key words: Borage seeds oil; $\gamma$-irradiation; Hepatotoxicity; Antioxidant.

Abbreviations: GLA, $\gamma$-linolenic acid; BO, Borage oil; GC-MS, Gas chromatography-Mass Spectrometry; MPa, Megapascal (Unit of pressure); Gy, The Gray (derived unit of ionizing radiation dose); AST, Aspartate aminotransferase; ALT, Alanine aminotransferase; GGT, Gamma glutamyl transferase; GSH, Reduced glutathione; TG, Triacylglycerols; TC, Total cholesterol; LDL-C, Low density lipoprotein cholesterol; HDL-C, High density lipoprotein cholesterol; MDA, Lipid peroxide (Malondialdehyde); ROS, Reactive oxygen species; RT, Retention time.

\section{Introduction}

Ionizing radiation generally affects the living tissues by damaging the cell with direct and indirect action; direct process produces damage signal transduction, as well as disruption in the cells, while indirect effect results from its communication with water-soluble molecules and production of free radicals and their subsequent action on subcellular substrates that destroy the structure of cells (Devasagayam et al., 2004; Ebadi, 2007). Ionizing radiationinduces damage in hepatic tissues, as it is one of the most radiosensitive organs (Widmaier et al., 2004). Excess reactive oxygen species (ROS) damage cellular components and cause lesions in an organism. Activated oxygen, however, readily reacts with biomembrane polyunsaturated fatty acids to generated lipid peroxidation, which disturbs physiological functions of living cells (Sardesai, 1995).

Cells show an elaborate defense system to destroy these ROS, which are the antioxidant defenses. The imbalance between oxidant/antioxidants is leading to an excessive production of oxygen metabolites, which creates an oxidative stress (Ho et al., 1998). Antioxidants have the capacity to reduce harmful effects of radiation on normal tissues (Okunieff et al., 2008). Antioxidants supplements reduce the treatment-related adverse effects, which occur during radiation therapy by overcome the oxidative damage to normal cells (Lawenda et al., 2008).

The use of traditional plant-based preparations in remedial therapeutic and preventive medicines has been developed. Borage (Borago officinalis L.) from Boraginaceae family is an annual herbaceous plant with nutritional 


\section{Khattab et al., Afr J Tradit Complement Altern Med., (2017) 14 (4): 169-179 \\ https://doi.org/10.21010/ajtcam.v14i4.20}

value used in traditional medicine and culinary uses in some countries (Asadi-Samani et al., 2014; Ghahremanitamadon et al., 2014). Borage seeds oil contains mainly triacylglycerol (95\%) consisting of C16-C20 fatty acids, while 5\% presents minor components composed of tocopherols, flavonoids, phospholipids, sterols, free fatty acids and also mono-and diacylglycerols (Kotnik et al., 2006). Several classes of these components act as antioxidants (Shahidi and Shukla, 1996). Borage seeds oil is the richest plant source of $\gamma$-linolenic acid (GLA) which is used as dietary supplement for prevention and/or treatment of various degenerative pathologies illnesses (Asadi-Samani et al., 2014), as osteoporosis (Kruger et al., 1998), diabetes (Das, 2010), and cancer (Ge et al., 2009 ; Itoh et al., 2010). GLA has also been reported to suppress tumor growth in vitro (Pham et al., 2006), improve oxygenation status (Mancuso et al., 1997), exert anti-inflammatory activity and display beneficial effects in the early stages of sepsis (Horrobin, 1992; Pontes-Arruda et al., 2011). Therefore, this study aimed to identify and determine fatty acids composition of the oil extracted from borage seeds, as well as to evaluate the potential radioprotective effect of BO against whole body $\gamma$ irradiation- induced hepatotoxicity in male rats.

\section{Materials and Methods \\ Plant Material}

Borage seeds (Borago officinalis L.) were obtained from Medicinal and Aromatic Plants Research Department, Horticultural Research Institute, Agricultural Research Center, Ministry of Agriculture. Seeds were authenticated by Dr. Abdel-Halim Abdel-Mogly Mohamed, Senior researcher of Taxonomy, Flora and Phytotaxonomy Researchers Department, Horticultural Research Institute, Agricultural Research Center, Giza, Egypt.

\section{Animals}

Male Albino rats (160-180 g) were obtained from Animal Breeding Unit of the National Research Center (NRC). Rats were kept in standard laboratory conditions. Experiment was done in National Center for Radiation Research and Technology (NCRRT), Atomic Energy Authority, Egypt. Animals were carefully handled in accordance with the guidelines of Committee for Care and Use of Laboratory Animals, National Research Center, Dokki, Egypt.

\section{Radiation Source}

Whole body gamma irradiation (6.5 Gy) was performed according to Naguib and Abd El Magaid (2007) at NCRRT, Atomic Energy Authority, Cairo, Egypt, using Gamma Cells-40 biological irradiator furnished with a Caesium-137. The radiation dose rate was $0.61 \mathrm{~Gy} / \mathrm{min}$. as calibrated at the time of the experiment.

\section{Extraction of Oil from Borage Seeds}

Dry borage seeds $(750 \mathrm{~g})$ were crushed using a commercial blender and then subjected to cold pressing. In this method, oil was extracted by continuous screw-pressers (Carver Press, USA) without any chemicals for 20 min at a pressure of $49.0 \mathrm{MPa}$.

\section{Fatty Acids Composition. Preparation of Fatty Acids Methyl Esters}

Sulphuric acid $(30 \mathrm{ml})$ and absolute methanol (4: $96 \mathrm{~V} / \mathrm{V})$ were mixed with $0.2 \mathrm{~g} \mathrm{BO}$, then heated under reflux for about 3 hours. The methyl esters were extracted thrice with petroleum ether, and washed several times with distilled water till the washings were neutral to phenolphthalein. The combined fatty acids methyl esters layers were dried over anhydrous sodium sulphate and filtered. The ether was then removed using a rotary evaporator and aliquots of the fatty acid methyl esters were analyzed by mass spectrometer (Ludde et al., 1960).

\section{Gas Chromatography-Mass Spectrometry Analysis of Fatty Acids Methyl Esters}

Gas Chromatography-Mass Spectrometry (GC-MS) analysis of fatty acids methyl esters of BO was performed according to Adams (1995) with a Finnigan Mat SSQ 7000 gas chromatography coupled to Flame ionization detector (FID). The analysis was conducted under programmed temperature conditions: $140-240{ }^{\circ} \mathrm{C}$ at $5{ }^{\circ} \mathrm{C}$ min-1 in 30 min (detector and injector temperatures of $260{ }^{\circ} \mathrm{C}$ ), injection volume and mode of $0.4 \mu \mathrm{L}$ and split (100:1), and nitrogen gas as carrier gas $(20 \mathrm{~cm}$ min-1). The desired amount of samples was dissolved in $2 \mathrm{~mL}$ of hexane before injection. The flow-rate of $1 \mathrm{~mL} \mathrm{~min}-1$ with isocratic elution of acetonitrile for $34 \mathrm{~min}$. Compounds of the different fatty acids were identified by their retention times (RT) and interpretation of their mass spectra. 


\section{Experimental Design}

Rats ( $\mathrm{n}=60)$ were divided into five groups (12 rats/group), all groups fed the standard AIN-93 diet (Reeves et al., 1993), and given water ad libitum. The following scheme explained the experimental design: Control group; rats (nonirradiated and non-treated) received orally $0.5 \mathrm{ml}$ of distilled water with two drops of Tween-80, Irradiated group (Irrad); rats were exposed to single sub-lethal dose $(6.5 \mathrm{~Gy})$ of whole body $\gamma$-radiation and received orally $0.5 \mathrm{ml}$ of distilled water with two drops of Tween-80, Borage oil group (BO); rats (non-irradiated) received $50 \mathrm{mg} / \mathrm{kg}$ b.wt $/$ day BO (Hamed and Wahid, 2015), through an intragastric gavage dissolved in $0.5 \mathrm{ml}$ of distilled water with two drops of Tween-80. Irradiated BO post-treated group; rats received the same gavage dosage of BO orally started 3 hours after irradiation and continued daily for 2 weeks. Irradiated BO prepost-treated group; rats were administered orally with BO (50 mg $/ \mathrm{kg} \mathrm{b.wt)} \mathrm{daily,} \mathrm{started} \mathrm{one} \mathrm{week} \mathrm{before} \mathrm{irradiation} \mathrm{and} \mathrm{continued} \mathrm{till} \mathrm{the} \mathrm{end} \mathrm{of} \mathrm{the} \mathrm{experiment} \mathrm{(} 2$ weeks after irradiation). Six rats from each group were sacrificed at the interval of 7 and 15 days post irradiation. Serum samples were collected for biochemical analysis and liver samples for biochemical and histopathological examinations.

\section{Assay of Biomarkers of Hepatotoxicity and Lipids Profile}

Serum alanine aminotransferase (ALT), aspartate aminotransferase (AST) (Reitman and Frankel, 1957), gamma glutamyl transferase (GGT) (Szewczuk et al., 1988), and lipids profile parameters; triacylglycerols (TG) (Fossati and Prencipe, 1982), total cholesterol (TC) (Meiattini et al., 1987), high density lipoprotein cholesterol (HDL-C) (Demacker et al., 1980), and low density lipoprotein cholesterol (LDL-C) (Marchall, 1992) were determined.

\section{Assay of Serum and Hepatic Reduced Glutathione (GSH) and Lipid Peroxide (MDA)}

After blood collection, rats of each group were sacrificed under anesthesia, the liver samples were removed immediately, washed twice with ice-cold phosphate buffer saline (PBS) and homogenized in 5 volume of phosphate buffer ( $\mathrm{pH} 8.0,0.01 \mathrm{M}$ ) using a Polytron homogenizer (pt 3100) (five cycles of $10 \mathrm{~s}$ at $3000 \mathrm{rpm}$ ). Aliquots were prepared according to Arafa et al. (2005), and used for the assessment of GSH and MDA. Serum and hepatic GSH levels were determined according to Jollow et al. (1974). The procedure is based on the reaction of bis-(3-carboxy-4nitrophenyl) disulfide reagent by SH group to form 2-nitro-5-mercaptobenzoic acid, which has an intense yellow color that was measured spectrophotometrically at $412 \mathrm{~nm}$. Serum and hepatic lipid peroxide levels were measured by the methods of Vashney and Kale (1990). The procedure involved the reaction between malondialdehyde (MDA; endproduct of lipid peroxidation) and thiobarbituric acid to yield stable pink chromospheres, which measured spectrophotometrically at $532 \mathrm{~nm}$.

\section{Histopathological Examination}

Liver samples of the sacrificed rats were fixed immediately in $10 \%$ neutral formalin solution. The fixed specimens were trimmed, washed and dehydrated in ascending grades of alcohol. The specimens were sectioned at 4-6 microns thickness and stained with Hematoxylin and Eosin (H\&E stain) then examined under ordinary microscope according to Bancroft et al. (1996).

\section{Statistical Analysis}

Results were statistically analyzed using SPSS version 22, one-Way ANOVA, Post Hoc, LSD to compare between groups in the same time interval.

\section{Results \\ Fatty Acids Composition}

The identification of individual fatty acids and percentages were carried out by GC-MS and presented in Table (1). The peaks related to different fatty acids at different retention times (RT) were shown in Figure (1), some of these peaks were detected and identified, while others about (3.43\%) were unidentified. Results in Table (1) showed that 11 fatty acids were identified and detected in the oil extracted from borage seeds, some of these fatty acids were saturated represented (13.26\% of total fatty acids) as Lauric, palmitic, stearic and arachidic acid, and the most predominant saturated fatty acid was palmitic acid (7.64\%). Seven unsaturated fatty acids were detected and identified including palmitoleic, oleic, linoleic, $\gamma$-linolenic, brassidic, erucic and nervonic acids, total unsaturated fatty acids represented ( $83.31 \%$ of total fatty acids composition). The unsaturated linoleic acid represented the majority of total fatty acids composition (34.23\%) followed by $\gamma$-linolenic (24.79\%) and oleic acid (14.23\%). 
Khattab et al., Afr J Tradit Complement Altern Med., (2017) 14 (4): 169-179

https://doi.org/10.21010/ajtcam.v14i4.20

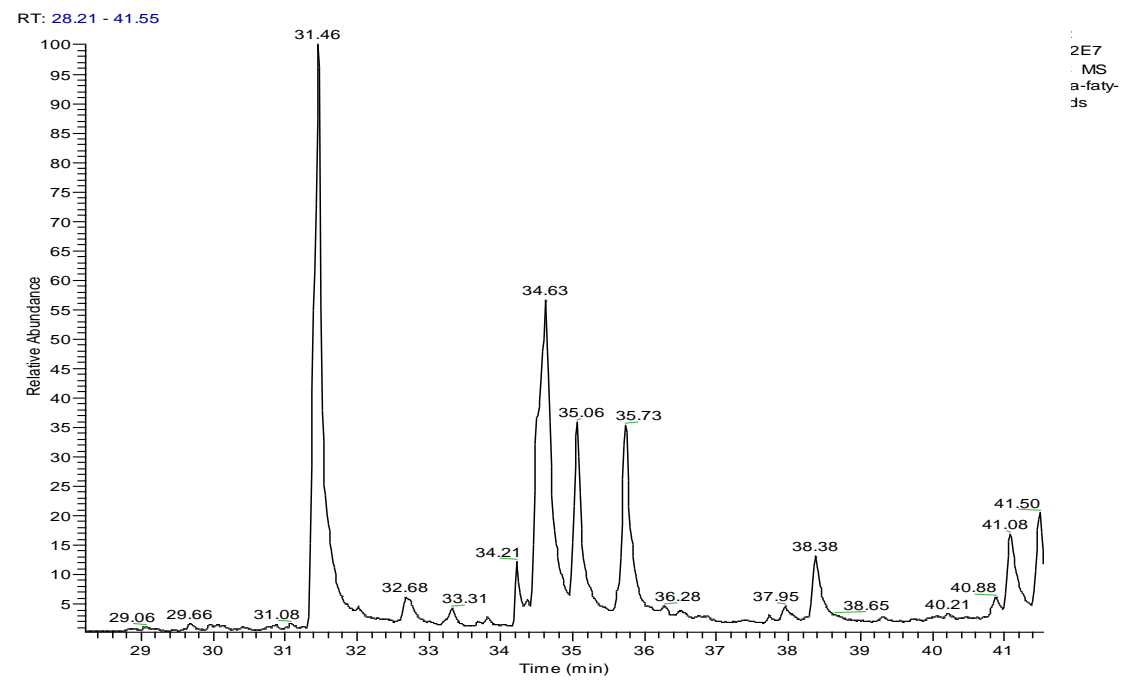

Figure 1: GC-MS of fatty acids methyl esters of oil extracted from borage seeds

Table 1: Fatty acids methyl esters composition of BO as a percentage concentrations of total fatty acids.

\begin{tabular}{|l|l|l|l|}
\hline Common Name & RT $(\min )$ & \% & Systematic Name \\
\hline Lauric acid & 29.06 & 1.14 & Dodecanoic acid \\
\hline Palmitic acid & 29.66 & 7.64 & Hexadecanoic acid \\
\hline Palmitoleic acid $*$ & 30.05 & 6.25 & Cis-9- Hexadecanoic acid \\
\hline$U$ & 30.40 & 1.55 & \\
\hline Stearic acid & 30.86 & 3.08 & Octadecanoic acid \\
\hline Oleic acid * & 31.08 & 14.23 & Cis-9-Octadecanoic acid \\
\hline U & 31.46 & 0.15 & \\
\hline Linoleic acid $*$ & 32.68 & 34.23 & Cis-9,12-Octadecanoic acid \\
\hline$\gamma$ - Linolenic acid $*$ & 34.21 & 24.79 & Cis-9,12,15-Octadecanoic acid \\
\hline Brassidic acid $*$ & 36.28 & 0.06 & Trans-13- Docosenoic acid \\
\hline Arachidic acid & 37.40 & 1.4 & Eicosanoic acid \\
\hline U & 38.38 & 1.73 & \\
\hline Erucic acid $*$ & 2.06 & Cis-13-docosenoic acid \\
\hline Nervonic acid* & 40.46 & 1.69 & Cis-15-Tetracosenoic acid \\
\hline Unsaturated fatty acids & 41.08 & $83.31 \%$ of total fatty acids \\
\hline Saturated fatty acids & $13.26 \%$ of total fatty acids \\
\hline
\end{tabular}

*: Unsaturated fatty acid.

$\mathrm{U}$ : undetected fatty acid methyl ester.

\section{Effect of BO on Some Biomarkers of Hepatotoxicity}

Data presented in Table (2) showed that exposure of rats to 6.5 Gy of whole body $\gamma$-radiation induced a significant increase $(\mathrm{p}<0.001)$ in the activities of AST, ALT and GGT in irradiated rats compared to the corresponding levels in non-irradiated control rats at the $7^{\text {th }}$ and $15^{\text {th }}$ days post radiation exposure. While both irradiated BO post and preposttreated groups showed significant amelioration in the serum levels of ALT, AST and GGT when compared to the corresponding irradiated group, there were significant decrease $(\mathrm{p}<0.001)$ at the $15^{\text {th }}$ day when compared with irradiated group. It was found that, BO prepost-treatment had more powerful effect against irradiation induced hepatotoxicity than BO post-treatment. 
Khattab et al., Afr J Tradit Complement Altern Med., (2017) 14 (4): 169-179

https://doi.org/10.21010/ajtcam.v14i4.20

Table 2: Effect of BO on serum transaminases (AST\&ALT) and gamma glutamyl transferase (GGT) levels in irradiated (Irrad) rats

\begin{tabular}{|c|c|c|c|c|c|c|}
\hline \multirow[b]{2}{*}{ Parameters } & \multirow{2}{*}{$\begin{array}{c}\text { Time } \\
\text { intervals } \\
\text { (Day) }\end{array}$} & \multicolumn{5}{|c|}{ Experimental groups } \\
\hline & & Control & Irrad & BO & $\begin{array}{c}\text { Irrad BO } \\
\text { post-treated }\end{array}$ & $\begin{array}{c}\text { Irrad BO } \\
\text { prepost-treated }\end{array}$ \\
\hline \multirow{4}{*}{$\begin{array}{c}\mathbf{A S T} \\
(\mathrm{U} / \mathrm{L})\end{array}$} & \multirow{2}{*}{$7^{\text {th }}$} & & $\mathrm{a}^{* * * * *}$ & $\mathrm{~b}^{* * * *}$ & $\mathrm{a}^{* *} \mathrm{~b}^{* *} \mathrm{c}^{* * *}$ & $a^{*} b^{* *} c^{* *}$ \\
\hline & & $108.98 \pm 9.85$ & $138.12 \pm 12.65$ & $106.22 \pm 9.28$ & $122.59 \pm 9.49$ & $120.92 \pm 7.11$ \\
\hline & & & $a^{* * * *}$ & $\mathrm{~b}^{* * * *}$ & \multirow{2}{*}{$\begin{array}{c}a^{*} b^{* * * *} c^{*} \\
117.93 \pm 10.5 \\
8 \\
\end{array}$} & $\mathrm{~b}^{* * *}$ \\
\hline & $15^{\text {th }}$ & $106.97 \pm 9.64$ & $136.45 \pm 11.40$ & $104.56 \pm 8.50$ & & $115.09 \pm 9.49$ \\
\hline \multirow{4}{*}{$\begin{array}{l}\text { ALT } \\
(\mathrm{U} / \mathrm{L})\end{array}$} & & \multirow{2}{*}{$41.04 \pm 2.59$} & $\mathrm{a}^{* * *}$ & $\overline{b^{* * * *}}$ & $a^{* *} b^{*} c^{* *}$ & $a^{*} b^{* *}$ \\
\hline & $7^{\text {th }}$ & & $54.56 \pm 5.35$ & $41.03 \pm 4.01$ & $49.76 \pm 4.69$ & $46.61 \pm 3.64$ \\
\hline & \multirow[b]{2}{*}{$15^{\text {th }}$} & \multirow{2}{*}{$42.03 \pm 4.21$} & $\mathrm{a}^{* * * *}$ & $\mathrm{~b}^{* * * *}$ & $\mathrm{a}^{*} \mathrm{~b}^{* * *} \mathrm{c}^{*}$ & $\mathrm{~b}^{* * * *}$ \\
\hline & & & $53.72 \pm 4.89$ & $41.20 \pm 4.15$ & $46.94 \pm 4.06$ & $45.77 \pm 3.73$ \\
\hline \multirow{4}{*}{$\begin{array}{l}\text { GGT } \\
(\mathrm{U} / \mathrm{L})\end{array}$} & & & $a^{* * * *}$ & $\mathrm{~b}^{* * * *}$ & $\mathrm{a}^{* * * * b^{* * * *} \mathrm{c}^{*}}$ & $\mathrm{~b}^{* * * *}$ \\
\hline & $7^{\text {th }}$ & $4.43 \pm 0.38$ & $9.24 \pm 1.33$ & $4.7 \pm 0.76$ & $6.45 \pm 0.99$ & $4.58 \pm 0.77$ \\
\hline & & & $\mathrm{a}^{* * * *}$ & $\mathrm{~b}^{* * * *}$ & $\mathrm{~b}^{* * *}$ & $\mathrm{~b}^{* * * *}$ \\
\hline & $15^{\text {th }}$ & $4.33 \pm 0.33$ & $8.74 \pm 0.9$ & $5.00 \pm 0.52$ & $5.19 \pm 0.82$ & $4.58 \pm 0.77$ \\
\hline
\end{tabular}

Values were represented as means \pm SD $(n=6)$.

Statistical analysis was done between groups in the same time interval.

${ }^{a}$ Significant difference between control and irradiated groups. ${ }^{\mathrm{b}}$ Significant difference between irradiated and irradiated BO post and prepost-treated groups. ${ }^{c}$ Significant difference between non-irradiated BO and irradiated BO post and prepost-treated groups. ${ }^{\mathrm{d}}$ Significant difference between irradiated BO post-treated and irradiated BO prepost-treated groups. ( ${ }^{*} \mathrm{p}<0.05,{ }^{* *} \mathrm{p}<0.01$ and $\left.{ }^{* * *} \mathrm{p}<0.001\right)$

\section{Effect of BO on Reduced Glutathione (GSH) and Lipid Peroxide (MDA) in Irradiated Rats}

In irradiated group, serum and hepatic GSH activities were significantly reduced at both the $7^{\text {th }}$ and $15^{\text {th }}$ days post irradiation as compared with their corresponding levels in control group. However, irradiated BO post-treated and prepost-treated groups showed significant increase in GSH activities during the experimental periods ( $7^{\text {th }}$ and $15^{\text {th }}$ days) as compared with irradiated group. The hepatic and serum levels of MDA in irradiated rats showed significant increase at the $7^{\text {th }}$ and $15^{\text {th }}$ days compared with their corresponding levels in control group. However, both irradiated BO posttreated and prepost-treated groups induced significant improvement in the serum and hepatic MDA when compared with their levels in irradiated group at the $7^{\text {th }}$ and $15^{\text {th }}$ days post irradiation Tables (3\&4).

\section{Effect of BO on Lipids Profile of Irradiated Rats}

Table (5) showed the effect of $\mathrm{BO}$ on the serum levels of lipids profile of irradiated rats. In irradiated group the levels of TG, TC, and LDL-C revealed a significant increase $(\mathrm{p}<0.001)$, while HDL-C level recorded a significant decrease $(\mathrm{p}<0.001)$ at the $7^{\text {th }}$ and $15^{\text {th }}$ days post irradiation as compared to the corresponding values in control rats. Lipids profile parameters were significantly improved in both irradiated BO post-treated and prepost-treated groups as compared with irradiated group. The improvement was more pronounced in irradiated $\mathrm{BO}$ prepost-treated group than irradiated BO post-treated group.

Table 3: Effect of BO on serum reduced glutathione (GSH) and lipid peroxide (MDA) levels in irradiated (Irrad) rats

\begin{tabular}{|c|c|c|c|c|c|c|}
\hline \multirow[b]{2}{*}{ Parameters } & \multirow{2}{*}{$\begin{array}{c}\text { Time } \\
\text { intervals } \\
\text { (Day) }\end{array}$} & \multicolumn{5}{|c|}{ Experimental groups } \\
\hline & & Control & Irrad & BO & $\begin{array}{c}\text { Irrad BO } \\
\text { post-treated }\end{array}$ & $\begin{array}{c}\text { Irrad BO prepost- } \\
\text { treated }\end{array}$ \\
\hline \multirow{5}{*}{$\begin{array}{l}\text { GSH } \\
(\mathrm{mg} / \mathrm{dl})\end{array}$} & & & $\mathrm{a}^{* * * *}$ & $\mathrm{~b}^{* * * *}$ & $\mathrm{a}^{* *} \mathrm{~b}^{* * *} \mathrm{c}^{*}$ & $\mathrm{a}^{*} \mathrm{~b}^{* * * *} \mathrm{c}^{*}$ \\
\hline & $7^{\text {th }}$ & $68.34 \pm 6.59$ & $32.33 \pm 6.33$ & $70.13 \pm 5.47$ & $54.45 \pm 4.15$ & $57.95 \pm 10.38$ \\
\hline & & & $a^{* * * *}$ & $\mathrm{~b}^{* * * *}$ & $\mathrm{~b}^{* * * *}$ & $\mathrm{~b}^{* * * * *}$ \\
\hline & $15^{\text {th }}$ & $68.02 \pm 6.94$ & $36.23 \pm 5.90$ & $69.06 \pm 5.63$ & $60.22 \pm 6.73$ & $63.32 \pm 5.86$ \\
\hline & & & $\mathrm{a}^{* * * *}$ & $\mathrm{~b}^{* * * *}$ & $\mathrm{a}^{* *} \mathrm{~b}^{* * * * c^{*}}$ & $\mathrm{~b}^{* * * *}$ \\
\hline \multirow{3}{*}{$\underset{(\mathrm{nmol} / \mathrm{ml})}{\operatorname{MDA}}$} & $7^{\text {th }}$ & $73.04 \pm 7.01$ & $135.22 \pm 9.6$ & $69.54 \pm 6.21$ & $88.14 \pm 8.27$ & $70.64 \pm 6.17$ \\
\hline & & & $\mathrm{a}^{* * * *}$ & $\mathrm{~b}^{* * * *}$ & $\mathrm{~b}^{* * * *} \mathrm{c}^{*}$ & $\mathrm{~b}^{* * * *}$ \\
\hline & $15^{\text {th }}$ & $72.53 \pm 6.69$ & $126.32 \pm 7.18$ & $70.82 \pm 6.15$ & $79.52 \pm 7.83$ & $69.48 \pm 5.47$ \\
\hline
\end{tabular}

Values were represented as means \pm SD $(n=6)$.

Statistical analysis was done between groups in the same time interval. ${ }^{\text {a }}$ Significant difference between control and irradiated groups. ${ }^{\mathrm{b}}$ Significant difference between irradiated and irradiated BO post and prepost-treated groups. ${ }^{\mathrm{c}}$ Significant difference between non-irradiated BO and irradiated BO post and prepost-treated groups. ${ }^{\mathrm{d}}$ Significant difference between irradiated BO post-treated and irradiated BO prepost-treated groups. $\left({ }^{*} \mathrm{p}<0.05,{ }^{* *} \mathrm{p}<0.01\right.$ and ${ }^{* * *} \mathrm{p}<$ $0.001)$ 
Khattab et al., Afr J Tradit Complement Altern Med., (2017) 14 (4): 169-179

https://doi.org/10.21010/ajtcam.v14i4.20

Table 4: Effect of BO on hepatic reduced glutathione (GSH) and lipid peroxide (MDA) levels in irradiated (Irrad) rats

\begin{tabular}{|c|c|c|c|c|c|c|}
\hline \multirow[b]{2}{*}{ Parameters } & \multirow{2}{*}{$\begin{array}{c}\text { Time } \\
\text { intervals } \\
\text { (Day) }\end{array}$} & \multicolumn{5}{|c|}{ Experimental groups } \\
\hline & & Control & Irrad & BO & $\begin{array}{l}\text { Irrad BO post- } \\
\text { treated }\end{array}$ & $\begin{array}{c}\text { Irrad BO prepost- } \\
\text { treated }\end{array}$ \\
\hline \multirow{4}{*}{$\begin{array}{c}\text { GSH } \\
\text { (mg/g tissue) }\end{array}$} & & & $\mathrm{a}^{\text {***** }}$ & $\mathrm{b}^{* * * *}$ & $\mathrm{a}^{* * *} \mathrm{~b}^{* * * *} \mathrm{c} * * *$ & $\mathrm{a}^{*} \mathrm{~b} * * * * \mathrm{c}^{*} * *$ \\
\hline & $7^{\text {th }}$ & $49.97 \pm 4.65$ & $31.15 \pm 3.03$ & $51.99 \pm 4.75$ & $42.88 \pm 4.49$ & $43.72 \pm 4.49$ \\
\hline & & & $\mathrm{a}^{* * * *}$ & $\mathrm{~b}^{* * * *}$ & $\mathrm{a}^{*} \mathrm{~b}^{* * * *} \mathrm{c}^{*}$ & $\mathrm{~b}^{* * * *}$ \\
\hline & $15^{\text {th }}$ & $49.64 \pm 4.31$ & $32.65 \pm 2.82$ & $50.33 \pm 4.88$ & $44.05 \pm 4.42$ & $45.38 \pm 4.51$ \\
\hline \multirow{4}{*}{$\begin{array}{c}\text { MDA } \\
\text { (nmol/g tissue) }\end{array}$} & & & $\mathrm{a}^{* * * *}$ & $\mathrm{~b}^{* * * *}$ & $\mathrm{a}^{*} \mathrm{~b} \mathrm{~b}^{* *} \mathrm{c} * *$ & $\mathrm{a}^{*} \mathrm{~b}^{* * * *} \mathrm{c}^{*}$ \\
\hline & $7^{\text {th }}$ & $163.24 \pm 9.49$ & $192.22 \pm 11.65$ & $161.18 \pm 6.90$ & $175.43 \pm 6.74$ & $174.10 \pm 9.89$ \\
\hline & & & $\mathrm{a}^{* * * *}$ & $\mathrm{~b}^{* * * *}$ & $\mathrm{~b}^{* * * *} \mathrm{c}^{*}$ & $\mathrm{~b}^{* * * * *}$ \\
\hline & $15^{\text {th }}$ & $164.35 \pm 8.16$ & $188.56 \pm 10.28$ & $159.34 \pm 6.11$ & $171.10 \pm 9.12$ & $168.43 \pm 6.24$ \\
\hline
\end{tabular}

Values were represented as means $\pm \mathrm{SD}(\mathrm{n}=6)$.

Statistical analysis was done between groups in the same time interval. ${ }^{a}$ Significant difference between control and irradiated groups. ${ }^{\mathrm{b}}$ Significant difference between irradiated and irradiated BO post and prepost-treated groups. ${ }^{\mathrm{c}}$ Significant difference between non-irradiated BO and irradiated BO post and prepost-treated groups. ${ }^{\mathrm{d}}$ Significant difference between irradiated BO post-treated and irradiated BO prepost-treated groups. $\left({ }^{*} \mathrm{p}<0.05,{ }^{* *} \mathrm{p}<0.01\right.$ and ${ }^{* * *} \mathrm{p}<$ $0.001)$

Table 5: Effect of BO on serum lipids profile parameters in irradiated (Irrad) rats

\begin{tabular}{|c|c|c|c|c|c|c|}
\hline \multirow[b]{2}{*}{ Parameters } & \multirow[b]{2}{*}{$\begin{array}{c}\text { Time intervals } \\
\text { (Day) }\end{array}$} & \multicolumn{5}{|c|}{ Experimental Groups } \\
\hline & & Control & Irrad & BO & $\begin{array}{l}\text { Irrad BO post- } \\
\text { treated }\end{array}$ & $\begin{array}{c}\text { Irrad BO } \\
\text { prepost-treated }\end{array}$ \\
\hline \multirow{5}{*}{$\begin{array}{c}\text { TG } \\
(\mathrm{mg} / \mathrm{dl}) \\
\end{array}$} & & & $a^{* * * *}$ & $\mathrm{~b}^{* * * *}$ & $\mathrm{a}^{*} \mathrm{~b}^{* * * *} \mathrm{c}^{*}$ & $\mathrm{~b}^{* * * * c^{*}}$ \\
\hline & $7^{\text {th }}$ & $91.79 \pm 6.92$ & $146.85 \pm 15.33$ & $89.41 \pm 7.21$ & $104.04 \pm 7.75$ & $100.88 \pm 5.97$ \\
\hline & & & $\mathrm{a}^{* * * *}$ & $\mathrm{~b}^{* * * *}$ & $\mathrm{~b}^{* * * *}$ & $\mathrm{~b}^{* * *}$ \\
\hline & $15^{\text {th }}$ & $93.80 \pm 7.83$ & $136.77 \pm 10.04$ & $90.41 \pm 8.43$ & $100.99 \pm 8.3$ & $97.94 \pm 8.51$ \\
\hline & & & $\mathrm{a}^{* * * *}$ & $\mathrm{~b}^{* * * *}$ & $\mathrm{a}^{* * * * \mathrm{~b}^{* * * *} \mathrm{c}^{*}}$ & $\mathrm{a}^{* *} \mathrm{~b}^{* * * *} \mathrm{c}^{*}$ \\
\hline \multirow{3}{*}{$\underset{(\mathrm{mg} / \mathrm{dl})}{\mathbf{T C}}$} & $7^{\text {th }}$ & $87.12 \pm 4.29$ & $151.09 \pm 10.39$ & $88.46 \pm 4.47$ & $104.72 \pm 11.83$ & $98.38 \pm 7.95$ \\
\hline & & & $\mathrm{a}^{* * * * *}$ & $\mathrm{~b}^{* * * *}$ & $\mathrm{~b}^{* * * *}$ & $\mathrm{~b}^{* * * *}$ \\
\hline & $15^{\text {th }}$ & $89.72 \pm 6.90$ & $140.09 \pm 8.24$ & $89.45 \pm 6.51$ & $98.13 \pm 5.68$ & $95.32 \pm 6.11$ \\
\hline \multirow{4}{*}{$\begin{array}{l}\text { HDL-C } \\
(\mathrm{mg} / \mathrm{dl})\end{array}$} & & & $\mathrm{a}^{* * * *}$ & $\mathrm{~b}^{* * * *}$ & $\mathrm{~b}^{* * * *} \mathrm{c}^{*}$ & $\mathrm{~b}^{* * * *}$ \\
\hline & $7^{\text {th }}$ & $42.34 \pm 6.56$ & $24.12 \pm 4.5$ & $45.48 \pm 3.87$ & $39.22 \pm 3.27$ & $40.72 \pm 3.68$ \\
\hline & & & $\mathrm{a}^{* * * *}$ & $\mathrm{~b}^{* * * *}$ & $\mathrm{~b}^{* * * *}$ & $b^{* * * *}$ \\
\hline & $15^{\text {th }}$ & $43.27 \pm 6.69$ & $30.05 \pm 4.36$ & $44.43 \pm 3.71$ & $41.14 \pm 2.61$ & $43.69 \pm 6.07$ \\
\hline \multirow{4}{*}{$\begin{array}{l}\text { LDL-C } \\
(\mathrm{mg} / \mathrm{dl})\end{array}$} & & & $\mathrm{a}^{* * * *}$ & $\mathrm{~b}^{* * *}$ & $\mathrm{a}^{* * * * \mathrm{~b}^{* * * *} \mathrm{c}^{*}}$ & $\mathrm{a}^{* *} \mathrm{~b}^{* * * *} \mathrm{c}^{*} \mathrm{~d}^{*}$ \\
\hline & $7^{\text {th }}$ & $25.50 \pm 3.68$ & $97.51 \pm 7.11$ & $25.51 \pm 6.50$ & $44.54 \pm 9.37$ & $37.21 \pm 6.35$ \\
\hline & & & $\mathrm{a}^{* * * *}$ & $\mathrm{~b}^{* * *}$ & $\mathrm{~b}^{* * * *} \mathrm{c}^{*}$ & $\mathrm{~b}^{* * * *}$ \\
\hline & $15^{\text {th }}$ & $27.76 \pm 6.46$ & $82.51 \pm 8.23$ & $26.51 \pm 6.77$ & $35.55 \pm 7.62$ & $32.45 \pm 7.07$ \\
\hline
\end{tabular}

Values were represented as means $\pm \mathrm{SD}(\mathrm{n}=6)$.

Statistical analysis was done between groups in the same time interval. ${ }^{\text {a }}$ Significant difference between control and irradiated groups. ${ }^{\mathrm{b}}$ Significant difference between irradiated and irradiated BO post and prepost-treated groups. ${ }^{\mathrm{c}}$ Significant difference between non-irradiated BO and irradiated BO post and prepost-treated groups. ${ }^{\mathrm{d}}$ Significant difference between irradiated BO post-treated and irradiated BO prepost-treated groups. $\left({ }^{*} \mathrm{p}<0.05,{ }^{* *} \mathrm{p}<0.01\right.$ and ${ }^{* * *} \mathrm{p}<$ $0.001)$

\section{Histopathological Examination}

Microscopical examination of liver tissues from control rats showed normal histological structural of hepatic lobule (Fig 2(1)). Irradiation induced marked changes in liver tissues. Liver sections of irradiated rats showed hepatic necrosis area associated with leucocytic inflammatory cells at the $7^{\text {th }}$ day (Fig 2(2-a \&b)), while at the $15^{\text {th }}$ day showed focal hydropic degeneration of hepatocytes and portal infiltration with mononuclear inflammatory cells (Fig 2(3-a)), with focal perivascular leucocytic cells infiltration (Fig2 (3-b)). Liver sections in rats that were given BO revealed no histological alterations (Figs 3 (1)). In the irradiated group that was post-treated with BO liver sections showed congestion of hepatoportal blood vessels with portal infiltration at the $7^{\text {th }}$ day (Fig 3 (2)), while at the $15^{\text {th }}$ day, revealed normal histological structure (Fig 3 (3)). In the irradiated BO prepost-treated group, liver sections showed slight dilatation of hepatic sinusoids at the $7^{\text {th }}$ day (Fig $3(4)$ ), while at the $15^{\text {th }}$ day, examined sections revealed normal histological structure (Fig 3 (5)). 


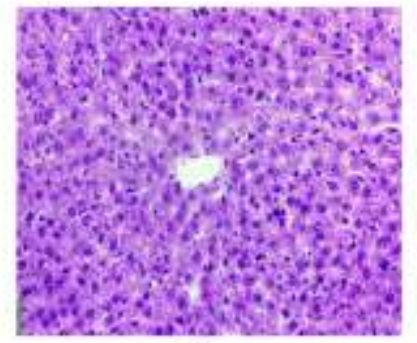

(1)

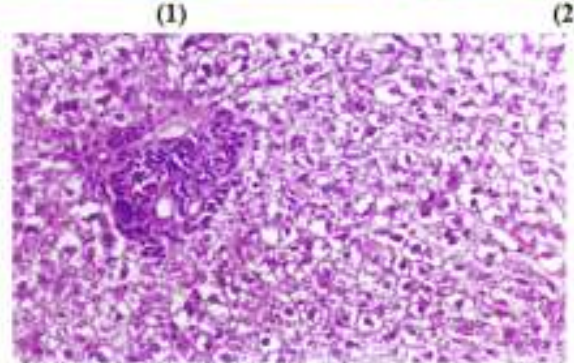

(3-a)

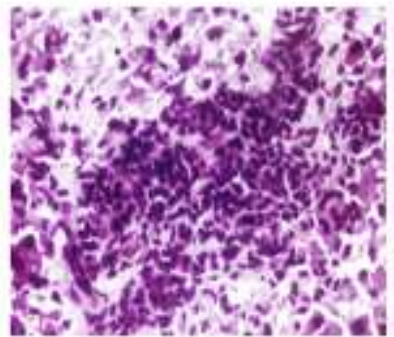

(2-a)

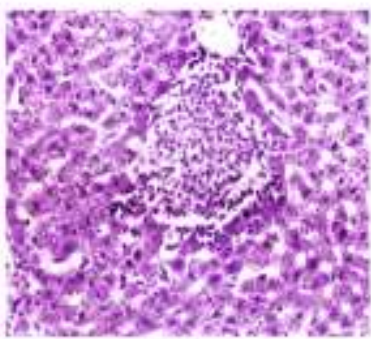

(2-b)

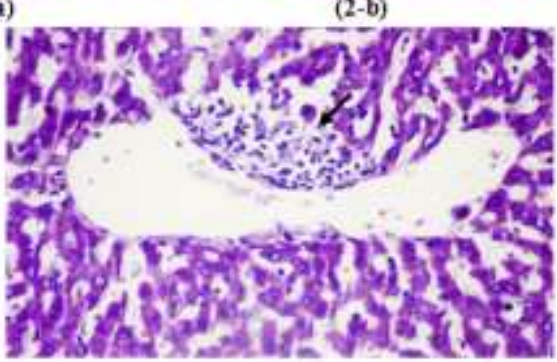

(3-b)

Figure 2: Liver sections from control showing normal structure (1). Liver sections of irradiated rats at the $7^{\text {th }}$ day showing hepatic necrosis area associated and replaced with leucocytic inflammatory cells ( 2 -a $\&$ b). while at the $15^{\text {th }}$ day showing focal hydropic degeneration of hepatocytes and portal infiltration with mononuclear inflammatory cells (3-a) (H \& E x 200), with focal perivascular leucocytic cells infiltration (arrow) (3-b) (H \& E x 400).

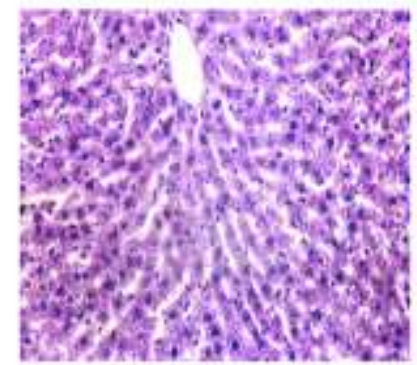

(1)

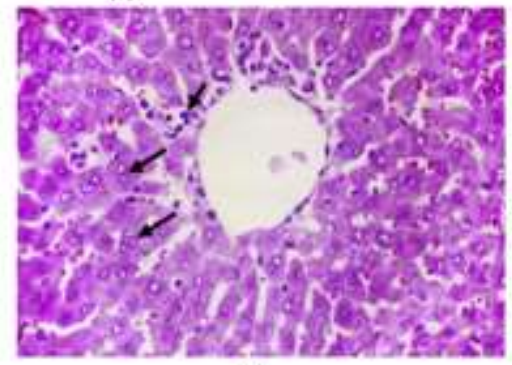

(4)

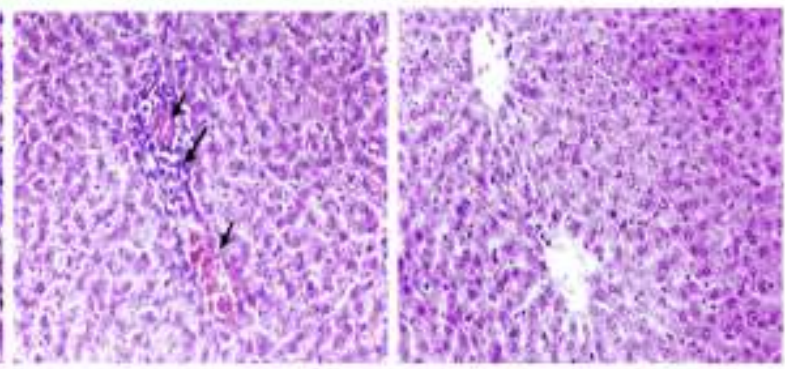

(2)

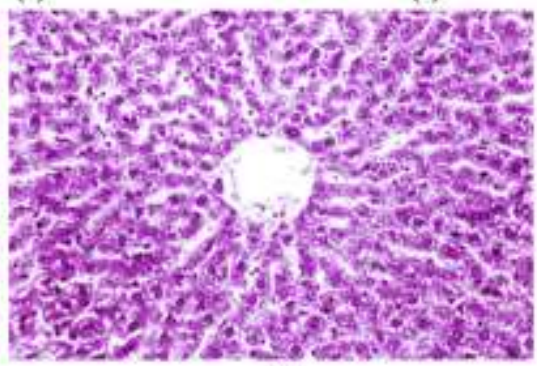

(5)

Figure 3: Liver from BO group showing no histopathological alterations (1). Liver sections of irradiated BO posttreated rats at the $7^{\text {th }}$ day showing hepatoportal blood vessels congestion (small arrows), as well as portal infiltration with mononuclear cells (large arrow) (2), while at the $15^{\text {th }}$ day showing apparent normal structure (3). Sections of irradiated BO prepost- treated rats at the $7^{\text {th }}$ day showing slight dilatation of hepatic sinusoids (small arrow) with few leucocytes in hepatic sinusoids and binucleation of hepayocytes (large arrows) (4) (H\&E x 400), while at the 15 ${ }^{\text {th }}$ day showing normal histological structure (5) (H \& E x 200).

\section{Discussion}

Reactive oxygen species (ROS) induce many changes in physiological and pathological states (Tawfik et al., 2006). They may be toxic through attacking the molecular level directly or indirectly by generating secondary reactive species (Brenneisen et al., 1997). These radicals may cause biomolecules oxidative damage (Kowaltowski and Vercesi, 


\section{Khattab et al., Afr J Tradit Complement Altern Med., (2017) 14 (4): 169-179 \\ https://doi.org/10.21010/ajtcam.v14i4.20}

1999). Borage oil is of great interest due to its high content of GLA (Bandoniene and Murkovic, 2002). This essential polyunsaturated fatty acid plays a role in health maintenance through involvement in cell membrane structure and synthesis of anti-inflammatory eicosanoids such as prostaglandins $E_{1}$ which have antioxidant and hepatoprotective properties (Lukivskaya et al., 2006). Borage seeds oil of the present study contained high percentage concentrations of linoleic $(34.23 \%)$ and $\gamma$-linolenic $(24.79 \%)$ acids. These fatty acids profile and concentrations are near to that reported in previous studies by Eskin (2008) and Tasset-Cuevas et al. (2013). Total unsaturated fatty acids (83.31\%) were higher than total unsaturated fatty acids (57.67\%) observed by El-Gengaihi et al. (2004) but similar to that observed by Khan and Shahidi (2000).

Liver enzymes and GGT are reliable markers of hepatotoxicity. The obtained data revealed significant increase in AST, ALT and GGT activities in irradiated rats throughout the experimental period. The observed increase in the hepatotoxicity biomarkers agree with the previous studies (Ashry and Hussein, 2007; Mansour and El-Kabany, 2009). The increase in the level of liver enzymes might be a result of the damage in hepatic cellular membranes (Gaur and Bhatia, 2009), impair of intrahepatic and extrahepatic bile flow, injury of hepatobiliary, or destruction of erythrocyte induced by irradiation (Singh et al., 2011). In this study, BO ameliorated $\gamma$-irradiation-induced hepatic injuries in rats. Our results agree with the previous findings showing that BO has hepatoprotective effect by reducing the production of pro-inflammatory mediators (Engler and Engler, 1998; Chen-Yang et al., 2014). Borage oil hepatoprotection is connected with its antioxidant properties (Lukivskaya et al., 2012). The phytochemical constituents, tocopherols and GLA are responsible for this hepatoprotective effect of borage seeds oil (Eskin, 2008; Soto et al., 2008).

The present results revealed significant acceleration in serum and hepatic MDA levels associated with depletion in serum and hepatic GSH levels due to radiation exposure. These results agree with, El-Dawy et al. (2007) and Mansour et al. (2014a) who reported that irradiation induced marked oxidative stress as evidenced by the significant increase in MDA with the significant decrease in GSH levels. The increase in MDA levels might be due to the interaction of free radicals with polyunsaturated fatty acids in the phospholipids portion of cellular membranes (Prasad et al., 2005). The decrease in GSH levels might be due to its consumption during the oxidative stress induced by ionizing radiation (Mansour, 2013). However, BO induced significant improvement in serum and hepatic antioxidant status. Previous studies indicated that borage seeds oil was one of the richest plant that contains $\gamma$-linolenic acid (Kotnik et al., 2006; Asadi-Samani et al., 2014). The high levels of GLA in BO might have accentuated the treating action of this oil, where $\gamma$-linolenic acid is needed for the synthesis of hormone-like prostaglandins, which regulate certain aspects of metabolism, protect against prooxidants induced membrane and cellular damage, and possess a modulatory action on the antioxidant status. Several studies evaluated the relationship between antioxidant activity of borage extract and its GLA content (De Haro et al., 2002; Del Río-Celestino et al., 2008). Tasset-Cuevas et al. (2013) have shown that both borage seed oil and GLA were able to desmutagenise the genotoxic activity of hydrogen peroxide by scavenging the ROS originated by the model genotoxicant used. The protective effect of BO on liver in this study can be related to its function of scavenging free radicals and to its high content of GLA.

Concerning lipids profile, the present study showed that irradiation induced hyperlipidemia in the form of significant increase in serum levels of TG, TC and LDL-C and decreased levels of HDL-C compared to the control group. These data are in agreement with earlier studies by Mansour (2013) and Mansour et al. (2014b). Several mechanisms have been postulated to explain such effects. The stimulation by gamma irradiation of liver enzymes responsible for the biosynthesis of fatty acids and mobilization of fat from adipose tissues to the blood stream leads to hyperlipidemic state (Darwish et al., 2007). Also, hormonal imbalance, in which the high insulin or the low glucagon level enhances the synthesis of triacylglycerol in both adipose tissues and liver, which is accompanied by the acceleration of fatty acids mobilization from the fat deposits to the blood (Baker et al., 2009). The amelioration of lipids parameters by BO may be attributed to $\gamma$-linolenic acid (prostaglandin precursor), which has been known to improve insulin-mediated glucose metabolism and abnormal lipids profile (Certik, 1993). The obtained results are in accordance with El-Gengaihi (2004) who found that administration of borage oil to rats led to a significant improvement in lipids parameters. In addition, Seman et al. (1999) found that GLA, as a content of borage oil, has great hypocholesterolemic ability in humans.

In the present study, irradiation produced damage reflected by deleterious changes in the structure of liver tissues. These results agree with Chen et al. (2001) and Soliman et al. (2007) who found that gamma irradiation induced injury, swelling, and pathological damage of rats' liver. The pretreatment with antioxidant agent prevents the injurious effect of irradiation on rats' liver. These findings may be due to the fact that exposure to radiation causes injury to blood vessels with degeneration and necrosis of hepatic parenchyma (Jirle et al., 1990). However, BO has a protective effect against morphological changes induced by $\gamma$-irradiation, and the improvement is more pronounced in both irradiated BO post-treated and prepost-treated groups at the $15^{\text {th }}$ day. This may be due to the fatty acids composition of BO, which promises the useful protector against the production of free radicals induced by gamma irradiation (Kawashima et al., 2002).

\section{Conclusion}

In conclusion, oral administration of BO, which is rich in $\gamma$-linolenic acid (GLA), significantly lower $\gamma$-irradiationinduced oxidative damage and hepatotoxicity in rats. The mechanisms of BO that provide protection against $\gamma$ - 


\section{Khattab et al., Afr J Tradit Complement Altern Med., (2017) 14 (4): 169-179 \\ https://doi.org/10.21010/ajtcam.v14i4.20}

irradiation-induced toxicity may be explained by its antioxidant activity, inhibition of MDA, and prevention against GSH depletion due to its high content of GLA. Therefore, BO may be used as a beneficial supplement for patients during radiotherapy treatment. and

\section{Acknowledgements}

This work was supported by the Deanship of Scientific Research (DSR), King Abdulaziz University, Jeddah, under grant No. (253-72-D1436). The authors, therefore, gratefully acknowledge the DSR technical and financial support.

The authors would like to thank Dr. Sherin S.E. Abo-Shanb, Medical and Radiation Research Department, Nuclear Materials Authority, Egypt for her help in the practical part of this study.

\section{References}

1. Adams, R.B. (1995). Identification of essential oil compounds by gas chromatography mass spectroscopy. Allured Pub. Corp., Carol. Stream, Illinois USA.

2. Arafa,H.M., Abd-Ellah,M.F. and Hafez,H.F. (2005). Abatement by naringenin of doxorubic-induced cardiac toxicity in rats. J. Egypt. Nat. cancer Inst., 17 (4): 291-300.

3. Asadi-Samani, M., Bahmani, M. and Rafieian-Kopaei, M. (2014). The chemical composition, botanical characteristic and biological activities of Borago officinalis: a review. Asian Pac J Trop Med., 7S1:S22-28. doi: 10.1016/S1995-7645(14)60199-1.

4. Ashry, O.M. and Hussein, E.M. (2007). Radioprotective potency of ginseng on some haematopoeitic and physiological parameters in irradiated rats. Egypt J. of Rad. Sci. Applic., 20(1):39-53.

5. Baker, J.E., Fish, B.L., Su, J., Haworth, S.T., Strande, J.L., Komorowski, R.A., Migrino, R.Q., Doppalapudi, A., Harmann, L., Allen, L.X., Hopewell, J.W. and Moulder, J.E. (2009). 10 Gy total body irradiation increases risk of coronary sclerosis, degeneration of heart structure and function in a rat model. Int. J. Radiat. Biol., 85 (12):10891100 .

6. Bancroft, D., Stevens, A. and Turmer, R. (1996). Theory and practice of histological technique, 4th ed., Churchill Living Stone, Edinburgh, London, Melbourne. p.47-67.

7. Bandoniene, D. and Murkovic, M. (2002). The detection of radical scavenging compounds in crude extract of borage (Borago officinalis L.) by using an on-line HPLC-DPPH method. J Biochem. Biophys. Method., 53(13):45-49.

8. Brenneisen, P., Briviba, K., Wlaschek, M., Wenk, J. and Scharffetter-Kochanek, K. (1997). Hydrogen peroxide increases the steady-state mRNA levels of collagenase/kkp-1 in human dermal fibroblasts. Free Rad. Bio. Med., 22(3):515-524.

9. Certik, M. (1993). Significance of gamma-linolenic acid at various pathological statues. Farm Obzor.,62:289292.

10. Chen, X., Hu, Y., Wang, Y., Yang, Z., Luo, X. and Sun, M. (2001). Protective effect of catecholomic acid derivatives on radiation-induced damage of rat liver mitochondria. Chinese J. Radiol. Med. Prot., 21(2):111- 113.

11. Chen-Yang, Y., Lu-Te, C., Wen-Cheng, H., Chien-Wei, H., Dz-Chi, C., Kee-Ching, G.J. and Ting-Yu, K. (2014). Preventive effects of borage oil and ling-zhi-8 protein on carbon tetrachloride-induced acute hepatic toxicity in rats. Curr. Topics in Nutraceutical Res., 12(3):91-99.

12. Darwish, M.M., Hussien, E.M. and Haggag, A.M. (2007). Possible role of licorice roots (glycyrrhiza glabra) as a natural radioprotector against oxidative damage in rats. Egypt. J. Rad. Sci. Applic., 20 (1):95-108.

13. Das, U.N. (2010). A defect in Delta6 and Delta5 desaturases may be a factor in the initiation and progression of insulin resistance, the metabolic syndrome and ischemic heart disease in South Asians. Lipids Health Dis., 9:130.

14. De Haro, A., del Río, M., Alcaide, B., Rapoport, H. and Cabrera, A. (2002). Characterisation and evaluation of species of the Boraginaceae family as source of gamma-linolenic acid for Mediterranean conditions in Proceedings of the $26^{\text {th }}$ International Horticultural Congress: The Future for Medicinal and Aromatic Plants, 629:231-237.

15. Del Río-Celestino, M., Font, R. and De Haro-Bailón, A. (2008). Distribution of fatty acids content in edible organs and seed fractions of borage (Borago officinalis L.). J. Sci. Food. Agric., 88(2):248-255.

16. Demacker, P.N.M., Vos-Janssen, H.E., Hifmas, A.G.M., Vants Lear, A. and Jansen, A.P. (1980). Measurement of high density lipoprotein cholesterol; in serum, comparison of sex isolation methods combined with enzymatic cholesterol analysis. Clin. Chem., 26: 1780.

17. Devasagayam, T.P., Tilak, J.C., Boloor, K.K., Ghaskadbi, K.S. and Lele, R.D. (2004). Free radicals and antioxidants in human health: Current status and future prospects. J. Assoc. Phys. India, 52: 794-804.

18. Ebadi, M. (2007). Pharmacodynamic basis of herbal medicine. Taylor and Francis group, LLC Boca Raton, London, New York, Washington, D.C.P., $2^{\text {nd }}$ ed., pp.485-493.

19. El-Dawy, H., Tawfik, S.S., El-Khafif, M. and Ragab, M.H. (2007). Role of taurine as a treatment for oxidative damage and sperm head abnormalities in irradiated mice and their male offspring. Egypt J Rad. Sci. and Applic., $20(1): 1-16$. 
20. El-Gengaihi, S.E., Salem, A., Bashandi, S.A., Ibrahim, N.A. and Abd El-Hamid, S.R. (2004). Hypolipidemic effect of some vegetable oils in rats. Food, Agriculture Envir., 2(2):88-93.

21. Engler, M.M. and Engler, M.B. (1998) Dietary borage oil alters plasma, hepatic and vascular tissue fatty-acid composition in spontaneously hypertensive rats. Prostaglandins, leukotriens and essential fatty acids, 59(1):11-15.

22. Eskin, N.A. (2008). Borage and evening primrose oil. Eur. J. Lipid Sci. Technol., 110: 651-654.

23. Fossati, P. and Prencipe, L. (1982). Serum triglycerides determined colorimetrically with an enzyme that produces hydrogen peroxide. Clin. Chem., 28 (10):2077-2088.

24. Gaur, A. and Bhatia, A.L. (2009). Modulation of phosphatase levels in mice liver by genistein treatment against radiation exposure. Pharmacognosy Res.,1(2):72-79.

25. Ge, H., Kong, X., Shi, L., Hou, L., Liu, Z. and Li, P. (2009). Gamma-linolenic acid induces apoptosis and lipid peroxidation in human chronic myelogenous leukemia K562 cells. Cell Biol. Int., 33(3):402-410.

26. Ghahremanitamadon, F., Shahidi, S., Zargooshnia, S., Nikkhah, A., Ranjbar, A. and Soleimani Asl, S. (2014). Protective effects of Borago officinalis extract on amyloid beta-peptide (25-35)-induced memory impairment in male rats: a behavioral study. Biomed. Res. Int. 2014:798535.

27. Hamed, A.N.E. and Wahid, A. (2015). Hepatoprotective activity of Borago officinalis extract against CCl4induced hepatotoxicity in rats. J. of Nat. Prod., 8:113-122.

28. Ho, Y.S., Magnenat, J.L., Cargano, M. and Cao, J. (1998). The nature of antioxidant defense mechanisms: a lesson from transgenic studies. Environ. Health Perspect., 106 (Suppl 5): 1219-1228.

29. Horrobin, D.F. (1992). Nutritional and medical importance of gamma-linolenic acid. Prog Lip Res., 31(2):163194.

30. Itoh, S., Taketomi, A., Harimoto, N., Tsujita, E., Rikimaru, T., Shirabe, K., Shimada, M. and Maehara, Y. (2010). Antineoplastic effects of gamma linolenic acid on hepatocellular carcinoma cell lines. J Clin Biochem Nutr., 47(1):81-90.

31. Jirle, R.L., Anschar, M.S. and Alati, T. (1990). Radiation sensitivity of the liver. Advances in Rad. Biol., 14:269311.

32. Jollow, G.L., Mitchell, J.R. and Zampaghone, N. (1974). Bromobenzene induced liver necrosis, protective role of glutathione and evidence for 3,4- bromobenzene oxide as the hepatotoxic metabolite. Pharm., 11(3):151-169.

33. Kawashima, A., Shimada, Y., Nagao, T., Ohara, A., Matsuhisa, A. and Tominaga, Y. (2002). Production of structured TAG rich in 1,3-dicarpryloyl-2- gamma-linolenoyl glycerol from borage oil. J. Am. Oil Chem. Societ., 79, (9): 871-877.

34. Khan, M.A. and Shahidi, F. (2000). Oxidative stability of stripped borage and evening primrose oils and their emulsions in water. JAOCS., 77 (9): 963-968.

35. Kotnik, P., Škerget, M. and Knez. Z. (2006). Kinetics of supercritical carbon dioxide extraction of borage and evening primrose seed oil. Eur. J. Lipid. Sci. Technol., 108(7):569-576. doi: 10.1002/ejlt.200600070.

36. Kowaltowski, A.J. and Vercesi, A.E. (1999). Mitochondrial damage induced by condition of oxidative stress. Free Rad Bio Med., 26 (3-4):463-471.

37. Kruger, M.C., Coetzer, H., Winter, R., Gericke, G. and Van Papendrop, D.H. (1998). Calcium, gamma linolenic acid and eicosapentaenoic acid supplementation in senile osteoporosis. Aging, 10(5):385-394.

38. Lawenda, B.D., Kelly, K.M., Ladas, E.J., Sagar, S.M., Vickers, A. and Blumberg, J.B. (2008). Should supplemental antioxidant administration be avoided during chemotherapy and radiation therapy? J. Natl. Cancer. Inst., 100(11):773-783.

39. Ludde, F.E., Barvord, R.A. and Reimenschnider, R.W. (1960). Direct conversion of lipid components to their fatty acid methyl ester. J. Am. Oil Chem. Soc., 37(9): 447-451.

40. Lukivskaya, O.Y., Naruta, E., Sadovnichy, V., Kirko, S. and Buko, V.U. (2012). Reversal of experimental ethanol-induced liver steatosis by borage oil. Phytother Res., 26(11):1626-1631. doi: 10.1002/ptr.4621.

41. Lukivskaya, O.Y., Zavodnik, L., Knas, M. and Buko, V. (2006). Antioxidant mechanism of hepatoprotection by ursodeoxycholic acid in experimental alcoholic steatohepatitis. Adv. Med. Sci., 51:54-59

42. Mancuso, P., Whelan, J., DeMichele, S.J., Snider, C.C., Guszcza, J.A., Claycombe, K.J., Smith, G.T., Gregory, T.J. and Karlstad, M.D. (1997). Effects of eicosapentaenoic and gamma-linolenic acid on lung permeability and alveolar macrophage eicosanoid synthesis in endotoxic rats. Crit. Care Med., 25:(3):523-532.

43. Mansour, H.H. (2013). Protective effect of ginseng against gamma-irradiation-induced oxidative stress and endothelial dysfunction in rats. EXCLI J.,12:766-777.

44. Mansour, H.H., Abd El Azeem, M.G. and Ismael, N.E. (2014a). Protective effect of Moringa oleifera on $\gamma$ radiation-induced hepatotoxicity and nephrotoxicity in rats. AJPCT., 2(4):495-508.

45. Mansour, H.H., Ismael, N.E. and Hafez, H.F. (2014b). Modulatory effect of Moringa oleifera against gammaradiation-induced oxidative stress in rats. Biomed. Aging Pathology., 4:265-272.

46. Mansour, S.Z. and El-Kabany, H. (2009). Effects of Fructus piperis long extract on fibrotic liver of gammairradiated rats. Chinese Med.,4:2 doi:10.1186/1749-8546-4-2.

47. Marchall, W.L. (1992). Clin. Chem., $2^{\text {nd }}$ ed., Gower Medical Publishing, U.K., 222-236.

48. Meiattini, F., Prencipe, L., Bardelli, F., Gianninl, G. and Tarli, P. (1978). The 4-hydroxybenzoate/4aminophenazone chromogenic system used in the enzymatic determination of serum cholesterol. Clin. Chem., 24 (12):2161-2165. 
49. Naguib, N.I. and Abd El Magaid,A. (2007). Protective role of acetylsalicylic acid (aspirin) against gamma irradiation-induced ophthalmic and histological changes in rat's eye. Egypt. J. Rad. Sci. Applic., 20 (1):215-230.

50. Okunieff, P., Swarts, S., Keng, P., Sun, W., Wang, W., Kim, J., Yang, S., Zhang, H., Liu, C., Williams, J.P., Huser, A.K. and Zhang, L. (2008). Antioxidants reduce consequences of radiation exposure. Adv. Exp. Med. Biol., 614:165-178.

51. Pham, H., Vang, K. and Ziboh, V.A. (2006). Dietary gamma-linolenate attenuates tumor growth in a rodent model of prostatic adenocarcinoma via suppression of elevated generation of PGE (2) and 5S-HETE. Prostaglandins Leukot. Essent. Fatty Acids, 74(4): 271-282.

52. Pontes-Arruda, A., Martins, L.F., de Lima, S.M., Isola, A.M., Toledo, D., Rezende, E., Maia, M. and Magnan, G.B. (2011). Enteral nutrition with eicosapentaenoic acid, gamma-linolenic acid and antioxidants in the early treatment of sepsis: results from a multicenter, prospective, randomized, double-blinded, controlled study: the INTERSEPT study. Crit. Care, 15(3): R144. doi: 10.1186/cc10267.

53. Prasad, N.R., Menon, V.P., Vasudev, V. and Pugalendi, K.V. (2005). Radioprotective effect of sesamol on $\gamma$ radiation induced DNA damage, lipid peroxidation and antioxidants levels in cultured human lymphocytes. Toxicol., 209(3):225-235.

54. Reitman, S. and Frankel, S. (1957). A colorimetric method for the determination of serum oxaloacetic and glutamic pyruvic transaminases. Am J. Clin. Pathol., 28 (1):56-63.

55. Reeves, P.G., Nielsen, F.H. and Fahey, G.C. (1993). AIN-93 Purified diets for laboratory rodents: Final report of the American Institute of Nutrition Ad Hoc Writing Committee on the Reformulation of the AIN-76. A Rodent diet. J. Nutr.,123:1939-1951.

56. Shahidi, F. and Shukla, V.K.S. (1996). Nontriacylglycerol constituents of fats and oils. INFORM., 7(11):12271232.

57. Sardesai, M. (1995). Role of antioxidants in health food. Nutr. Clin. Pract.,10 (1):19-55.

58. Seman, L.J., DeLuca, C., Jenner, J.L., Cupples, L.A., McNamara, J.R., Wilson, P.W., Castelli, W.P., Ordovas, J.M. and Schaefer, E.J. (1999). Lipoprotein (a)-cholesterol and coronary heart disease in the Framingham heart study. Clin. Chem., 45(7):1039-1046.

59. Singh, A., Bhat, T.K. and Sharma, O.P. (2011). Clinical biochemistry of hepatotoxicity. J. Clin. Toxicol., doi:10.4172/2161-0495.S4-001.

60. Soliman, O., Hafez, M.N. and Abd El Maguid, A. (2007). Histological and histochemical studies on liver of rats subjected to synergistic effect of natural and synthetic food colorants and/or gamma irradiation. Egypt J. Rad. Sci. Applic., 20 (1):195-214.

61. Soto, C., Concha, J. and Zuniga,M.E. (2008). Antioxidant content of oil and defatted meal obtained from borage seeds by an enzymatic-aided cold pressing process. Process Biochem., 43:696-699.

62. Szewczuk, A., Kuropatwa, M. and Lang, D. (1988). Colorimetric method for assay of serum gammaglutamyltransferase activity with some L-gamma- glutamyl-carboxyanilides. Clin. Chim. Acta., 178(1):35-40.

63. Tasset-Cuevas, I., Fernández-Bedmar, Z., Lozano-Baena, M.D., Campos-Sánchez, J., de Haro-Bailón, A., Muñoz-Serrano, A. and Alonso-Moraga, A. (2013). Protective effect of borage seed oil and gamma linolenic acid on DNA: in vivo and in vitro studies. PLOS One, 8 (2): e56986.

64. Tawfik, S.S., Abbady, M.I., Zahran, A.M. and Abouelalla, A.M.K. (2006). Therapeutic efficacy attained with thyme essential oil supplementation throughout $\gamma$-irradiated rats. Egypt J. Rad. Applic., 19 (1):1-22.

65. Vashney, R. and Kale, R.K. (1990). Effect of calmodulin antagonists on radiation-induced lipid peroxidation in microsomes. Int. J. Radiat. Biol., 58 (5):733-743.

66. Widmaier, E.P., Hershel, R. and Strang, K.T. (2004). Human physiology: the mechanisms of body functions, $9^{\text {th }}$ ed., Edited by Vander AJ, Sherman J and Iuciano DS., New York: McGraw Hill. 